\title{
CONTEXT-SENSITIVE EXTRACTION OF TREE CROWN OBJECTS IN URBAN AREAS USING VHR SATELLITE IMAGES
}

\author{
Juan Pablo Ardila, Valentyn A. Tolpekin, Wietske Bijker \\ Faculty of Geo-Information Science and Earth Observation (ITC) \\ University of Twente \\ 7500 AA Enschede, The Netherlands \\ ardila14710@itc.nl, tolpekin@itc.nl, bijker@itc.nl \\ http://www.boomenbeeld.nl
}

KEY WORDS: Object based image analysis, tree mapping, context classification, remote sensing urban areas, tree crown identification

\begin{abstract}
:
Municipalities need accurate and updated inventories of urban vegetation in order to manage green resources and estimate the profit of urban forestry activities. Earlier studies using high resolution satellite images have shown that automatic tree detection in urban areas using traditional classification techniques remains a very difficult task. This is mainly due to intra-crown spectral variation, heterogeneity of tree species and the complex spatial arrangement of individual trees with respect to other vegetated surfaces and elements of the urban space. This study aims to develop a reproducible object based image analysis (OBIA) methodology to locate and delineate individual tree crowns in urban areas using high resolution imagery and existing topographic maps. We propose an OBIA approach that considers the spectral, spatial and contextual characteristics of tree objects in the urban space. The classification strategy is implemented with classification rules that exploit object features at multiple segmentation levels such as spectral response, texture, size, geometry, roundness, and distance to shadow, which are used to modify the labeling and shape of image objects. The classification procedure was tested in a QuickBird image acquired over a city in The Netherlands with results indicating an identification rate of $75 \%$ for individual trees and a commission rate of $39 \%$. For the group of trees the identification rate was $100 \%$. We equally report on the geometrical and positional accuracy of the identified tree crown objects.
\end{abstract}

\section{INTRODUCTION}

Trees in urban areas represent a very important asset in densely built cities which leave few possibilities for the daily contact between man and nature. Multiple benefits of trees planted in urban areas have been described and reported (McHale et al., 2007, McPherson et al., 1994, Konijnendijk, 2005), mainly by forestry institutes and local authorities that are aware of the balance that should exist between urban infrastructure and green spaces. Those studies, have also stressed the relevance of monitoring the state of natural resources in cities. Since detailed inventories of the constantly changing urban green ecosystem are costly and difficult to update with traditional field survey methods, alternative solutions have been sought to harness the increasing amount of spatial data constantly captured over the Earth. This work, as part of the Boom en Beeld project in the Netherlands, aims to explore the use of object based image analysis (OBIA) and optical satellite imagery to implement a detailed tree crown inventory in urban areas.

Studies on implementation of detailed forest inventories with remote sensing imagery have indicated factors constraining the identification of tree crowns, such as the limited spatial resolution of spaceborne images, the increase of intra crown spectral variance found in very high resolution (VHR) imagery, and the low spectral separability between tree crowns and other vegetated surfaces in the understory (Gougeon and Leckie, 2006, Hirschmugl et al., 2007, Pouliot et al., 2002). In the urban environment, however, additional factors further complicate tree identification. In cities, trees spatially coexist with urban elements, such as buildings, roads, sidewalks and canals, which results in a complex arrangement of the space difficult to address in image interpretation. In addition, trees of different type and size may be found in strongly interlocked groups, evenly spaced or in arbitrary spatial patterns.

Considering that the mentioned factors limit the applicability of spectral classifiers to identify trees in images, a promising solution is to address the complexity of the urban space by introducing context information into the classification. Context information is fundamental for feature recognition and necessary to model our understanding of a spatial phenomena (Oliva and Torralba, 2007). For instance, context information for tree detection might be enforced in the classification with tuned conditions on the spatial distribution of the trees with respect to other elements of the urban infrastructure in the surroundings. Contextual rules can be used to model the occurrence of trees along roads, in private gardens or in green areas. In addition, depending on sun illumination at time of image acquisition, image shadows can be used to further guide tree identification. Similarly, geometric properties of tree crowns such as roundness and size can foster better identification results.

Recently, object based image analysis (OBIA) have gain popularity as a method bridging the gap between the increasing amount of detailed geospatial data and the inefficient results of conventional pixel based classifiers (Blaschke, 2010). OBIA has its ground on the processing and analysis of homogeneous regions and the consideration of the space formed by the aggregation of objects rather than individual pixels, which closely corresponds to the human vision process. Furthermore, OBIA recognizes that landscape features are evident at a wide range of scales and that those features are strongly related by their context.

In this work we develop OBIA methods for the identification of urban tree crown objects in VHR imagery. These algorithms interact according to the available context information and the type of tree objects in the image by exploiting the spectral, spatial and contextual characteristics of segmented image objects. We implement the OBIA approach in a QuickBird scene of the Bothoven district (Enschede, The Netherlands) and evaluate its performance using object based accuracy indicators. 


\section{EXPERIMENT DESIGN AND OBIA FOR TREE CROWN EXTRACTION}

The test site is a residential district of Enschede, The Netherlands, with an extension of 85 ha. The area has several tree species with a variation of tree inter-distance, crown size and surrounding elements that make it a very challenging and realistic benchmarking case for tree detection (Figure 1). In the study area, trees are planted along the roads, in green areas, and in private gardens, with crown diameter ranging from 1 to $25 \mathrm{~m}$. Depending on their location, surfaces surrounding trees are: pavement, grassland, and bare soil. As for distance between trees, the area contains isolated trees, tree pairs and clusters of interlocked trees. For this study we treat individual trees as isolated areas of tree crown with a diameter less than $20 \mathrm{~m}$ and group of trees as those areas exceeding this value. A total of 1140 individual trees and 44 groups of trees exist in the area (See section 2.6).

Tree detection was implemented in a Quickbird (QB) image captured on September 21, 2006 in multi-spectral and panchromatic mode with a spatial resolution of $2.4 \mathrm{~m}$ and $0.6 \mathrm{~m}$ respectively (Figure 1). The panchromatic mode captures surface reflectance between $0.4-0.9 \mu \mathrm{m}$, while the multi-spectral mode provides four spectral bands in the blue $(0.4-0.5 \mu \mathrm{m})$, green $(0.5-0.6 \mu \mathrm{m})$, red $(0.6-0.7 \mu \mathrm{m})$ and near infrared $(0.7-0.9 \mu \mathrm{m})$ parts of the electromagnetic spectrum. Sun elevation at time of image acquisition was $35^{\circ}$. OBIA for tree detection was performed after enhancing the spatial resolution of the image through a high pass kernel pan sharpening technique (Chavez et al., 1991), thereby mapping the $2.4 \mathrm{~m}$ multispectral bands to the $0.6 \mathrm{~m}$ of the panchromatic band. For information on urban infrastructure the digital topographic map of the Netherlands Top10NL (scale1:10.000) was used.

Given the varying spatial condition of trees in the area and the various factors limiting tree identification, we propose to divide the tree crown OBIA identification into tuned classification strategies that interact according to the image characteristics and the local context of the trees in the ground. In our approach, we first identify grassland areas and remove them from further analysis. Second, we detect isolated trees which present a good contrast with the background by constraining the spectral and geometric features of image objects. Third, a similar approach is used to identify trees which are obscured by shadows cast by tall buildings. Fourth, to identify very small young trees alongside the roads, we used shadow information and a road vector layer. Fifth, we identify the remaining trees with a local maxima filter and region growing according to shadow and geometric conditions. As a last step, we integrate the results of each classification strategy and generate a report of the extracted tree crown objects for individual and groups of trees.

OBIA tree detection was implemented in eCognition Developer 8 software (Definiens AG, 2009), using the multiresolution segmentation algorithm to generate image objects (Baatz and Schäpe, 2000) with modifications to address several scales of feature representation as explained below. Object features referenced here are described in the eCognition user guide(Definiens AG, 2009). The following sections present the modeled tree categories and the OBIA classification rules used for tree identification.

\subsection{Masking of grassland areas}

The identification of tree crown is largely affected by the spectral separability of tree crown pixels with respect to their background in the image. As vegetated surfaces such as grasslands and shrubs hinders the correct identification and delineation of tree crowns (Bunting and Lucas, 2006), we first identify grassland areas and

\begin{tabular}{|l|l|c|}
\hline Class & Feature & Value \\
\hline \hline Grass & Stdv NIR & $<50$ \\
& Area (m) & $>15$ \\
\hline Grass_neigh & Stdv NIR & $<60$ \\
& Area (m) & $>15$ \\
& Rel. border to Grass & $>0.3$ \\
& Length/Width & $>2.5$ \\
\hline
\end{tabular}

Table 1: Class description for identification of grassland areas

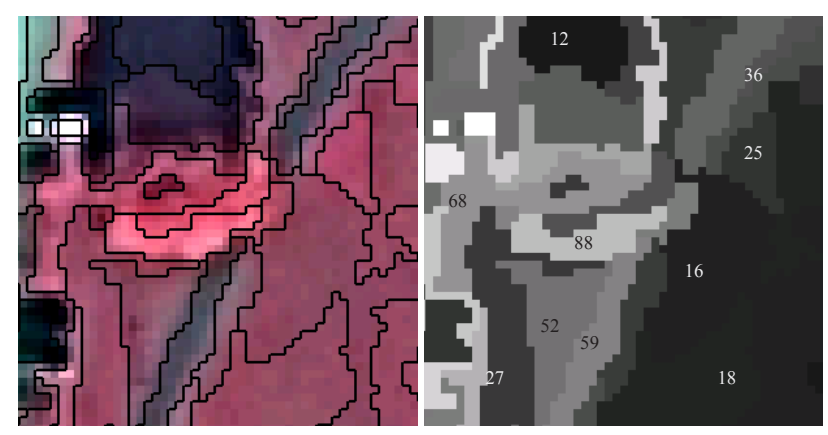

Figure 2: Object-wise Standard deviation for identification of grassland areas. (Left) false color multispectral composite $\operatorname{RGB}(4,3,2)$ with objects (black outlines) produced after segmentation with $p=25$; (Right) NIR standard deviation values for segmented objects

remove them from further analysis. We assist grassland identification by considering spectral and texture information values of image objects. Furthermore, as grassland areas occur at different scales, we implement a multi-level segmentation approach where objects are iteratively segmented with varying scale parameter $\left(p_{\text {seg }}\right)$ which decreases during each iteration step according to an update factor $\left(p_{\text {upd }} \epsilon(0,1)\right)$. Thus, the size of image objects decrease with each segmentation step until $p_{\text {seg }}$ reaches a defined minimum value. This procedure is presented in the following pseudocode:

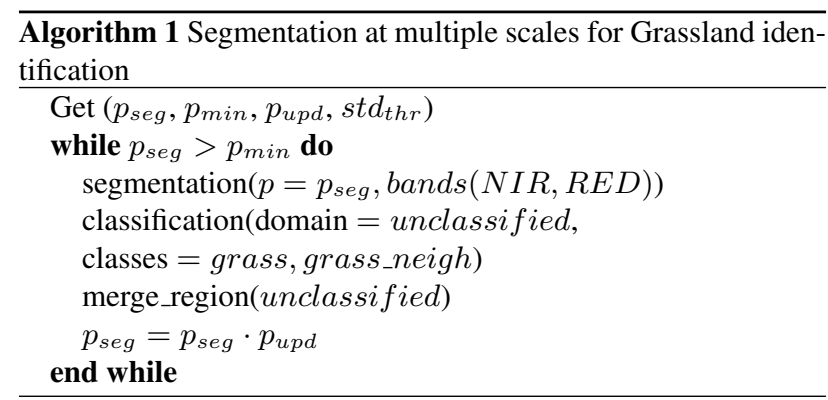

In this procedure, for each set of segmented objects with $p_{s e g}$, unclassified objects are assigned to Grass if their standard deviation in NIR is lower than a threshold $\left(s t d_{t h r}\right)$, and remain unclassified otherwise. For grassland identification in the area we used $p_{\text {seg }}=40, p_{\text {min }}=15$ and $p_{\text {upd }}=0.8$; while the selection of $s t d_{t h r}$ correspond to a value estimated from training set areas in the image. In addition, we define an additional class (Grass_neigh) to account for the edge texture effect of grassland regions. Examples of object standard deviation in the NIR band are shown in Figure 2. Table 1 presents the class description used to evaluate the object assignment to Grassland classes.

\subsection{Identification of medium and large trees with high back- ground contrast}

Once grassland areas are masked we classify trees which have a medium or good separability with respect to their background. 

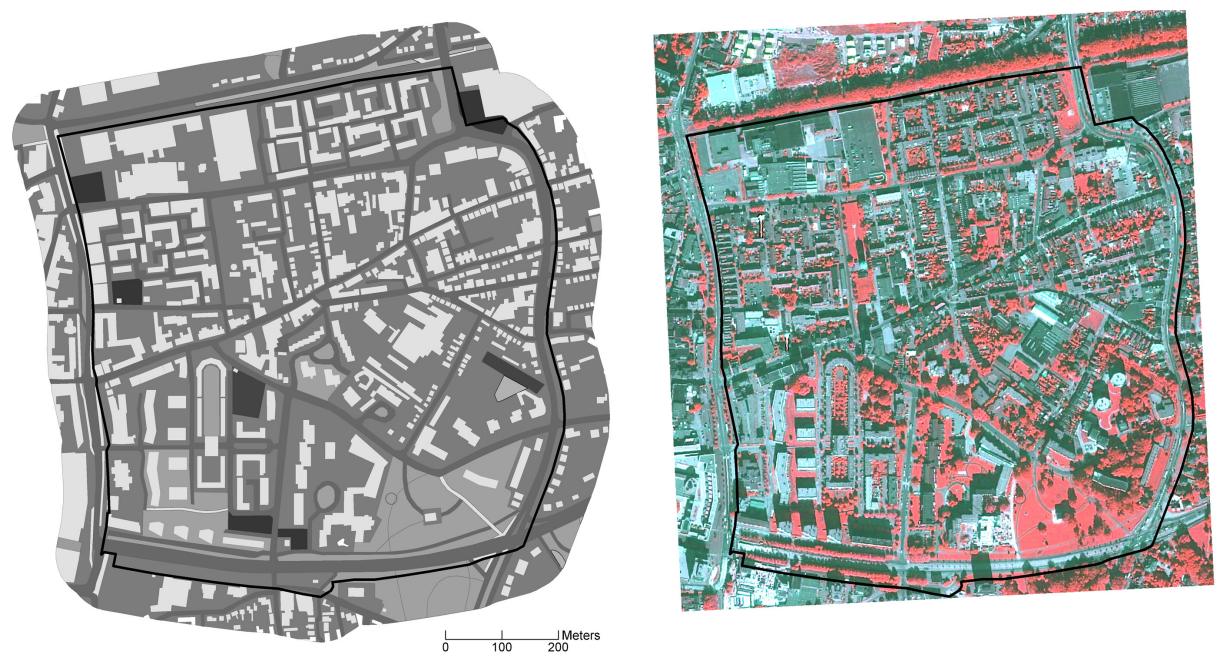

Figure 1: Bothoven district. Left: Top10NL digital map. Right: QB pan-sharpened band composition in false color multispectral $\operatorname{RGB}(4,3,2)$

\begin{tabular}{|l|l|l|}
\hline Algorithm & Object domain & Parameters \\
\hline \hline Chessboard segm. & Unclassified & Object size=1 \\
Classify & Unclassified & Class: candidate_TC \\
Merge region & $\begin{array}{l}\text { candidate_TC } \\
\text { classify }\end{array}$ & \\
\hline \hline Class & Feature & Value \\
\hline \hline candidate_TC & Mean (G) & $<500$ \\
& NDVI & $>140$ \\
\hline tree_crown & Area & $>6 m^{2}$ \\
& Length/width & $<1.3$ \\
& Roundness & $<0.5$ \\
& Std.dev (NIR) & $>40$ \\
\hline
\end{tabular}

Table 2: Algorithms and class description for identification of tree crowns with high background separability

Those trees, comprised by several pixels in the image, appear as single individuals which commonly stand in sealed surfaces such as pavement or concrete (Figure 3). To identify them, we compute a rescaled normalized vegetation index (NDVI) as shown in equation 1 and threshold it at the pixel level to separate vegetation pixels (candidate_TC in Table 2). Next, we identify tree crown objects (tree_crown in Table 2) by merging adjacent vegetation pixels and evaluating their class assignment according to their geometric properties. Table 2 presents the algorithms and classes used in this procedure.

$$
\mathrm{NDVI}=100\left(\frac{\mathrm{NIR}-\mathrm{R}}{\mathrm{NIR}+\mathrm{R}}+1\right)
$$

The use of the NDVI has the advantage that it is largely insensitive to intra-crown shadow variation resulting from sun illumination and physical structure of tree canopy. As seen in the radiometric profile of Figure 3, depending on the sun elevation angle at the time of image acquisition, one side of the crown is illuminated while the other is shaded in the visible and infrared part of the spectrum. Converserly, the NDVI is insensitive to this illumination effect. Notice also in Figure 3 that the profile shows a convex curve which is symmetric along the profile line. This property is exploited to generate tree crowns which have a circular and compact shape.

A second identification of tree crowns having regular shape and good background contrast is done by iteratively segmenting the

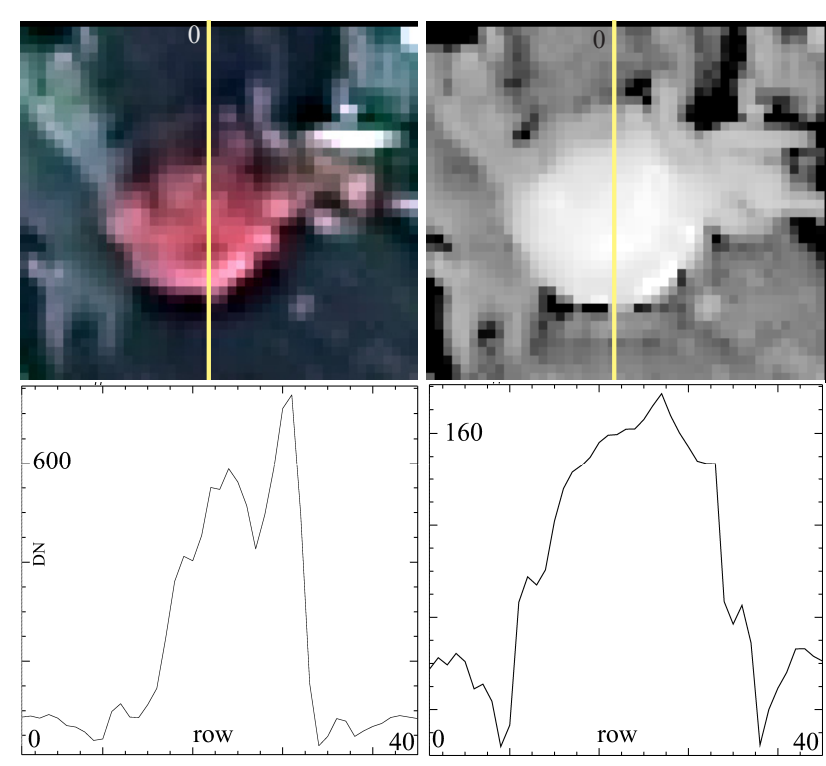

Figure 3: Tree crown with good radiometric background separability shown in false color composite $\operatorname{RGB}(4,3,2)$ (Top-left) and NDVI (Top-right) with their respective radiometric column profile along the yellow line for the infrared (bottom-right) and NDVI (bottom left)

image at multiple scales as defined in Algorithm 2 with parameters $p_{\text {seg }}=50, p_{\text {min }}=20$ and $p_{\text {upd }}=0.75$. For the set of objects generated at each segmentation step we asses the classification of tree crowns according to the geometric and spectral characteristics defined for tree_crown class in Table 2.

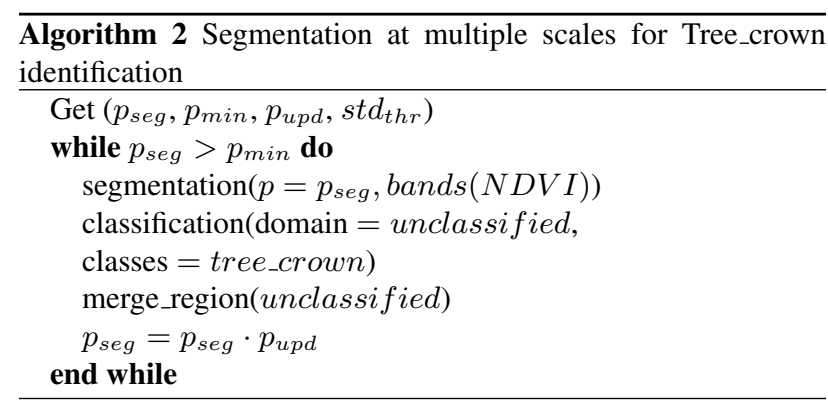




\begin{tabular}{|l|l|l|}
\hline Algorithm & Object domain & Parameters \\
\hline \hline Chessboard segm. & Unclassified & Object size=1 \\
Classify & Unclassified & Class: shadows \\
Merge region & shadows & \\
Classify & shadows & Class: shadows_tc \\
Local maxima & Unclassfied & NDVI with kernel=6 m; \\
& with roads=1 & Class:TC_max \\
Update variable & TC_max & TC_width = \\
& & $\Delta X$ (shadows_TC)@North \\
Classify & TC_max & Class: small_tree_crown \\
Grow region & small_tree_crown & Grow while \\
& & $\Delta X \leq T C \_w i d t h$ \\
\hline \hline Class & Feature & Value \\
\hline \hline shadows & Mean (Pan) & $<200$ \\
shadows_tc & Area & $>60 m^{2}$ \\
& Length/width & $<3$ \\
& Roundness & $<0.8$ \\
& Compactness & $<2$ \\
TC_max & Mean (NDVI) & $>50$ \\
small_tree_crown & TC_widht & $>0$ \\
\hline
\end{tabular}

Table 3: Algorithms and class description for identification of very small trees planted along the roads

\subsection{Identification of very small trees planted along roads}

A special case for detection are small trees which are either young or severely pruned each year, resulting in a crown which is smaller than the resolution cell size of VHR infrared satellite imagery. Examples of these trees are shown in Figure 4, where crown diameter is less than 3 meters for the shown trees. Due to sun illumination and reflectance of vegetation in the infrared, a local radiometric peak is found near the center of tree crowns (Gougeon and Leckie, 2006). We identify small trees on roads following a step-wise process, where we first detect tree locations using a local maxima filter in the NDVI and next we estimate the crown size according to the extent of the cast shadow on the ground. This classification strategy assumes that the sought tree objects are spatially isolated such that for each tree crown a single shadow object is detected through segmentation. Furthermore, this identification is constrained to the extent of road polygons of the topographic map.

As shown in Table 3 we identify shadows according to the spectral and geometric properties of segmented objects. Given the sun position at the time of image acquisition, shadows cast by trees are located on the north-side of the tree crowns in the image and have an elliptical shape. Those characteristics are used for identification of shadow_tc objects. As shown in the table, using the $\Delta X$ feature we measure the width of the shadow, which is then passed to the maxima objects (TC_max) as an estimation of the tree crown diameter. Next, if $\Delta X$ of the maxima objects (TC_max) is $>0$ the image pixels located at a distance $\leq \Delta X / 2$ are assigned to the individual tree crown.

\subsection{Identification of medium size trees}

The shadow relationship used for the identification of trees along the roads can also be used to identify a group of medium sized trees which do not have a clear contrast with the image background as those seen in Figure 5. Nonetheless, as the shadow cast by those trees often form a contiguous dark region in the image (Figure 5), a different strategy is adopted for the identification of tree shadows. We delineate individual tree crown shadow objects $\left(\right.$ shadow $_{t c}$ ) using an iterative segmentation procedure at multiple levels as proposed in Algorithm 2 and Table 3. After shadowtc objects are identified we search for local maxima in the domain of the unclassified objects as it was done for the detection of trees in roads. Likewise, we discard false maxima that

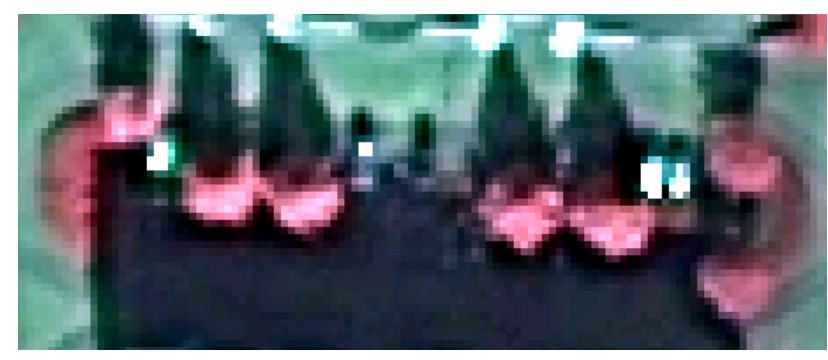

Figure 5: False color composite $\operatorname{RGB}(4,3,2)$ showing a group of trees with shadows cast by the trees and a building

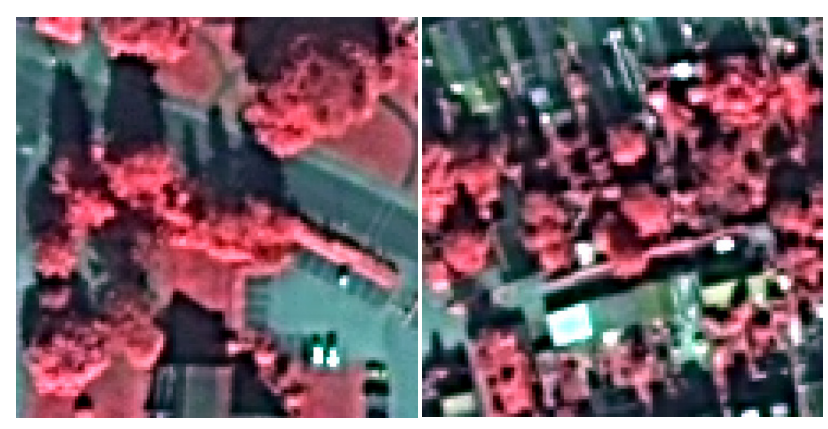

Figure 6: False color composite $\operatorname{RGB}(4,3,2)$ showing: (left) group of trees with large variation in size and species with presence of strong shadows in the image; (right) mixed group of trees and shrubs with different size and species planted in backyards

do not have shadowtc objects in the north and pass the $\Delta X$ attribute of the shadow objects to the local maxima objects so that the crown width can be estimated.

\subsection{Identification of individual trees with low background contrast}

Most of the trees in the study area are planted in clusters, very close to each other and with a strong variation in size and context. Identification of individuals is challenging for these trees due to the low spectral separability with the background and the presence of shadows cast by elements of the crown, neighboring trees or urban infrastructure. Furthermore, there is often a mixture of trees with shrub plants and hedges that complicate their identification. Examples of those trees are illustrated in Figure 6.

For identification of these trees we use the local maxima pixels as seeds which are the starting point of a region growing procedure aiming to delineate their crown (Gougeon and Leckie, 2006). In addition, to reduce the false positive detection we enforce the tree identification to the existence of shadows on the north-side of each local maxima within a certain distance. The region growing method starts with the identification of local maxima in the NIR band of the image. For each maxima object we allow the merging of adjacent pixels which are spectrally similar and represent a small difference in the variation of NIR and NDVI values respect to the digital value of the maxima. At each iteration new neighboring pixels are evaluated and merged to the tree crown provided the tree crown keeps a circular geometric shape. This procedure is explained in Algorithm 3.

Since the previous classification strategies aim to identify individual trees or small group of trees, we implemented an additional classification step to separate very large group of trees such as the one in the northern part of the area (see Figure 1). As those trees are spectrally identifiable and their objects do not have a definite 

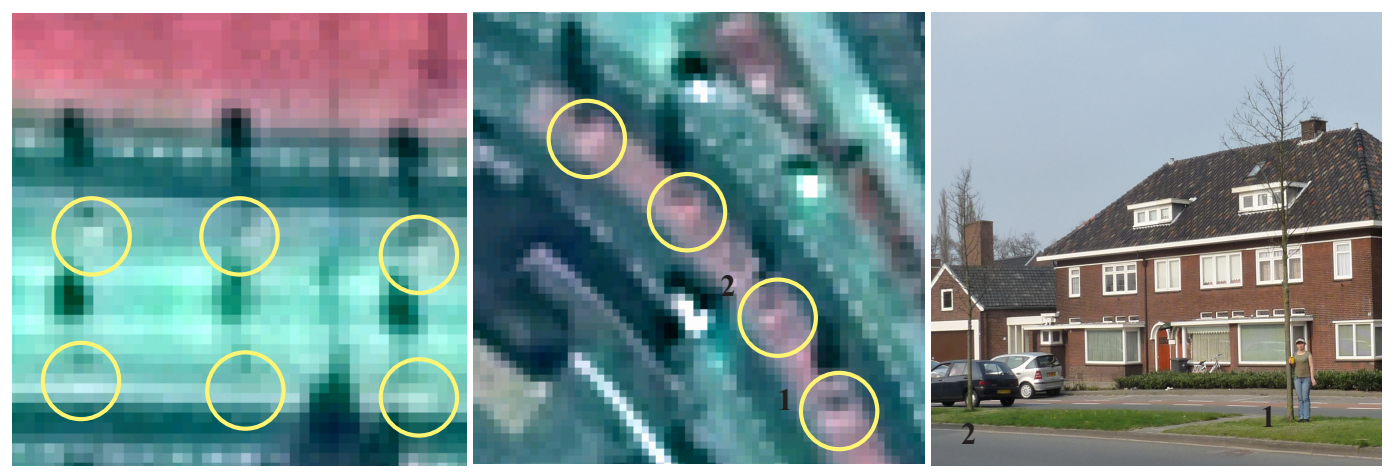

Figure 4: Small trees planted along the roads shown in false color composite RGB(4,3,2) (left and middle). Photograph of tree locations 1 and 2 in middle. For tree 1 crown diameter is $2.6 \mathrm{~m}$, height $5.9 \mathrm{~m}$ and stem diameter $10 \mathrm{~cm}$

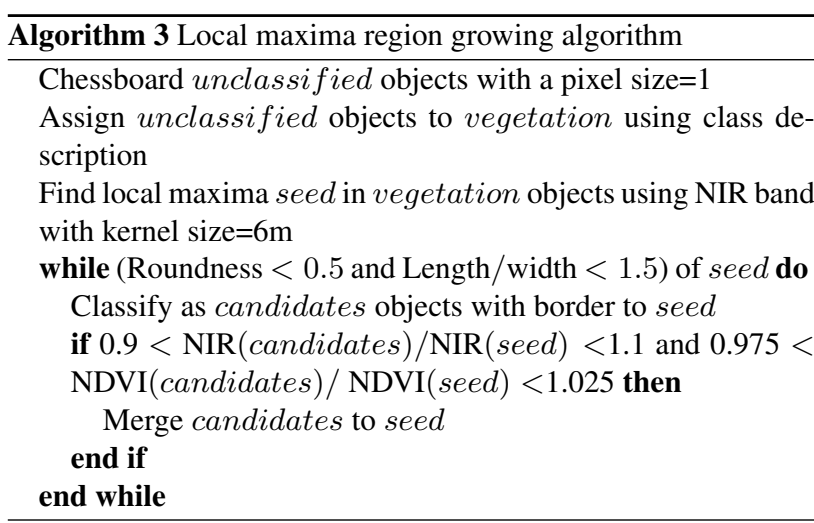

shape after segmentation, we used nearest neighbor classification in the object domain with class parameters taken from a set of training objects.

\subsection{Validation}

To validate the OBIA tree detection we construct a reference layer by digitizing tree crowns in the VHR aerial image 470258 of the DKLN Dutch dataset, acquired in summer 2006 with $40 \mathrm{~cm}$ resolution, supported by oblique images, field work and existing laser data of 2007. A first indication of We implemented object-based accuracy assessment, because the result in the form of tree crown objects is more meaningful for the users than the raster output. Our indicators of accuracy are based on polygon overlay between a classification and a reference layer and include commission errors (Type I) and omission errors (Type II) for tree identification. Furthermore, we adopt accuracy indicators related to the topological, geometrical and positional difference between the identified and reference tree crown objects. Although there are not clear methodologies for object based accuracy assessment we based ours in recent works (Möller et al., 2007, Clinton et al., 2008, Ragia and Winter, 2000) with some modifications. The topological accuracy quantifies how well the extent of classified objects fit the reference objects using measures of over and under identification of the extent of each tree crown object. The geometrical accuracy measures the positional correctness of the extracted objects based on the distance between the centroids of reference and classified tree objects.

\section{RESULTS}

The proposed OBIA methodology implemented in the Bothoven district identified 1253 individual trees and 46 groups of trees with a total of 1299 tree crown objects. As mentioned before, we consider individual trees those objects with a diameter less than $20 \mathrm{~m}$. Figure 7 shows OBIA results in six areas of the image according to the classification strategies defined in Section 2. With overlay analysis of reference data and OBIA results, we calculated the positive tree detections alongside with the errors type I (commission) and II (omission) as shown in Table 4.

\begin{tabular}{|l|l|l|l|}
\hline Type & Detection & Error Type I & Error Type II \\
\hline \hline Individual & 842 & $489(39 \%)$ & $298(26 \%)$ \\
Group & 44 & $2(5 \%)$ & $0(0 \%)$ \\
\hline
\end{tabular}

Table 4: Positive identification, and error types I (commission) and type II (omission) for individual trees and group of trees

From the table we observe that we identified 842 individual trees with an omission rate of $26 \%$ and a commission rate of $39 \%$. From this, $40 \%$ of the missing trees are very small individuals with area less than $5 \mathrm{~m}^{2}$ (tree crown diameter $<2.4 \mathrm{~m}$ ), which is the spatial resolution of the used multispectral images. Overdetection errors occurs mainly in private gardens and courtyards due to the presence of herbaceous and woody plants. We identified all groups of trees with an over identification of $5 \%$ and null omission errors.

The distribution of positional errors shown in Figure 8 reveals that most trees are correctly located within a radius of $2.2 \mathrm{~m}$. The scatter plot in Figure 9 presents the distribution of under and over detection errors for detected objects. As seen on the figure, most of the errors are under $40 \%$.

\section{DISCUSSION AND CONCLUSIONS}

With this study we have implemented an OBIA approach for detection of tree crowns in QB images. The approach took into consideration the different categories of trees according to their location in the city and their spatial, spectral and contextual characteristics. We implemented the method in a benchmarking area with a large variation of trees in size and location respect to their surroundings.

This methodology recognized the complex variation of trees in the urban space, and differs from previous work on tree extraction that often proposed a one-fits all strategy for tree identification that might serve well in forest plantations but is of limited application due the complexity and variability in cities. The OBIA approach implemented through classification rules permitted to address the spatial variability of trees in the city, which is revealed in classification strategies for different types of trees. The segmentation algorithms at multiple scales as it was tuned here, permit also to address the size variability of trees in cities, 

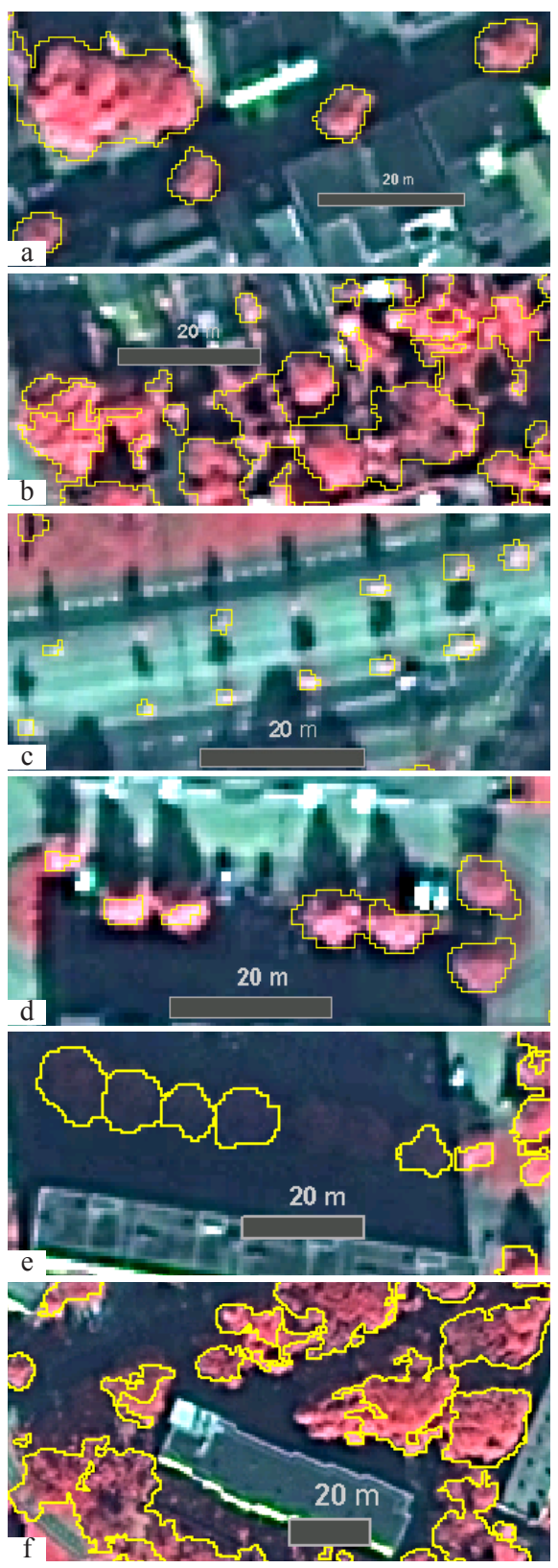

Figure 7: False color composite of QB Pan-sharpened with identified OBIA tree crown objects in yellow. a) Medium size and large trees with large background contrast; b) Individual trees with low background contrast; c) Very small trees planted along roads; d) Medium size trees; e) Trees in shadowed areas; f) Groups of trees

while the topological relationships of tree crown objects respect to shadows permitted to identify trees with not very clear spectral response.

The transferability of the classification rules defined here to other residential areas in the Netherlands and in images of different days or varying sun illumination remains to be tested. It is also attractive to test the OBIA analysis in images of higher resolution, such as the infrared digital DKLN aerial photographs of the Netherlands, acquired yearly. When doing this test, we should also assess the sensitivity of certain threshold used in the presented work.

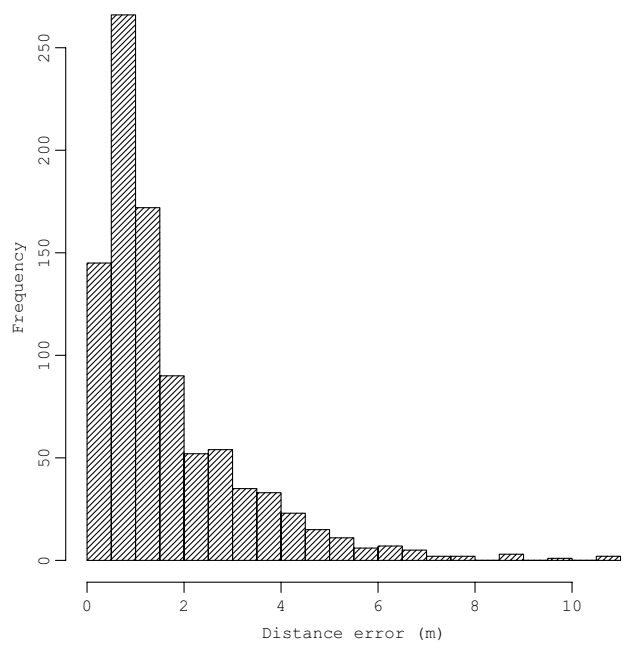

Figure 8: Distribution of positional accuracy errors for detected tree crowns

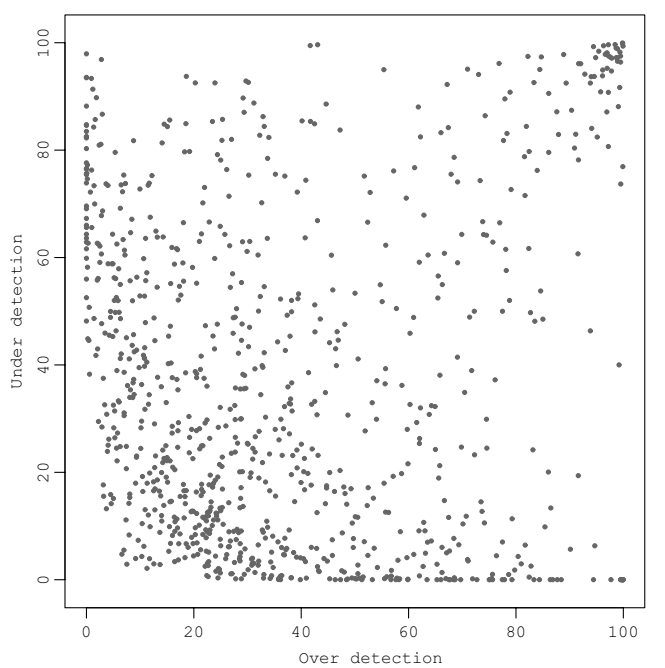

Figure 9: Distribution of over and under detection errors for positive tree crown identifications

\section{ACKNOWLEDGEMENTS}

This study has been made possible by funding of the Netherlands Space Office (NSO) through their program Pre-qualification ESA Programs (PEP) for the project Tree and Image (Boom en Beeld). Enschede municipality is kindly acknowledged for their contribution and supplying tree location data, topographic maps, cadastral data and aerial imagery. The Faculty of Geo-Information Science and Earth Observation (ITC) of the University of Twente is acknowledged for making the height data available.

\section{REFERENCES}

Baatz, M. and Schäpe, M., 2000. Multiresolution segmentation. an optimization approach for high quality multiscale image segmentation. In: J. Strobl, T. Blaschke and G.Griesebner (eds), Angewandte Geographische Informations-Verarbeitung XII, Karlsruhe, p. 1223.

Blaschke, T., 2010. Object based image analysis for remote sensing. Isprs Journal of Photogrammetry and Remote Sensing 65(1), pp. 2-16. 
Bunting, P. and Lucas, R., 2006. The delineation of tree crowns in australian mixed species forests using hyperspectral compact airborne spectrographic imager (CASI) data. Remote Sensing of Environment 101(2), pp. 230-248.

Chavez, P. S., Sides, S. C. and Anderson, J. A., 1991. Comparison of 3 different methods to merge multiresolution and multispectral data - LandSat TM and SPOT panchromatic. Photogrammetric Engineering and Remote Sensing 57(3), pp. 295-303.

Clinton, N., Holt, A., Gong, P. and Yan, L., 2008. An accuracy assessment measure for object based image segmentation. In: ISPRS (ed.), ISPRS Congress Beijing 2008, Vol. XXXVII, Beijing.

Definiens AG, 2009. Ecognition Developer, User guide. München.

Gougeon, F. A. and Leckie, D. G., 2006. The individual tree crown approach applied to Ikonos images of a coniferous plantation area. Photogrammetric Engineering and Remote Sensing 72(11), pp. 1287-1297.

Hirschmugl, M., Ofner, M., Raggam, J. and Schardt, M., 2007. Single tree detection in very high resolution remote sensing data. Remote Sensing of Environment 110(4), pp. 533-544.

Konijnendijk, C. C., 2005. Urban forests and trees : a reference book. Springer, Berlin; New York.

McHale, M. R., Gregory McPherson, E. and Burke, I. C., 2007. The potential of urban tree plantings to be cost effective in carbon credit markets. Urban Forestry \& Urban Greening 6(1), pp. 4960.

McPherson, E. G., Nowak, D. J. and Rowntree, R. A., 1994. Chicago's urban forest ecosystem: results of the Chicago urban forest climate project. Technical report, Department of Agriculture, Forest Service, Northeastern Forest Experiment Station.

Möller, M., Lymburner, L. and Volk, M., 2007. The comparison index: A tool for assessing the accuracy of image segmentation. International Journal of Applied Earth Observation and Geoinformation 9(3), pp. 311-321.

Oliva, A. and Torralba, A., 2007. The role of context in object recognition. Trends in Cognitive Sciences 11(12), pp. 520 - 527.

Pouliot, D. A., King, D. J., Bell, F. W. and Pitt, D. G., 2002. Automated tree crown detection and delineation in high-resolution digital camera imagery of coniferous forest regeneration. Remote Sensing of Environment 82(2-3), pp. 322-334.

Ragia, L. and Winter, S., 2000. Contributions to a quality description of areal objects in spatial data sets. ISPRS Journal of Photogrammetry and Remote Sensing 55(3), pp. 201-213. 